\title{
Smoking, asthma, chronic airflow obstruction and COPD
}

\author{
Malcolm R. Sears \\ Affiliation: McMaster University, Firestone Institute for Respiratory Health, St Joseph's Healthcare, Hamilton, \\ ON, Canada.
}

Correspondence: Malcolm R. Sears, McMaster University, Firestone Institute for Respiratory Health, St Joseph's Healthcare, 50 Charlton Avenue East, Hamilton, ON, L8N 4A6, Canada. E-mail: searsmamcmaster.ca

0 $@$ ERSpublications

Persistent asthma can lead to chronic airflow obstruction independently of smoking but smoking increases the risk http://ow.ly/GVfIJ

Cigarette smoking is the most prevalent risk factor for the development of adult-onset respiratory diseases associated with airflow obstruction, namely emphysema and chronic bronchitis, commonly called chronic obstructive pulmonary disease (COPD). Regrettably, childhood asthma does not reduce the likelihood of initiation of smoking [1] but it is not fully clear how smoking impacts pre-existing airway diseases such as asthma. Does smoking worsen asthma or superimpose another disease? How does asthma itself, irrespective of smoking, impact lung function in adulthood? What characteristics of asthma increase the risk of smoking-induced worsening of airflow obstruction? Does asthma lead to COPD?

Classically, the term COPD has been used for a cluster of diseases characterised by a combination of respiratory symptoms (cough, sputum production, dyspnoea and wheezing) with airflow limitation detected by spirometry, with incomplete reversibility. However, many studies of the prevalence of COPD conducted over the last decade have defined COPD physiologically, using a fixed cut-point for the post-bronchodilator forced expiratory volume in $1 \mathrm{~s}$ (FEV1)/forced vital capacity (FVC) ratio of 0.7 and an FEV1 below $80 \%$ of the predicted value to indicate airflow obstruction, without considering accompanying symptoms $[2,3]$.

The use of a fixed ratio for defining airflow obstruction has been strongly criticised $[4,5]$, as the normal ratio declines with increasing age and height even in healthy nonsmokers, resulting in up to $50 \%$ overdiagnosis above the age of 45 years. In contrast, in younger adults, the fixed ratio underestimates the presence of airflow obstruction [6]. Support for a revised definition of airflow obstruction based on age-specific lower limits of normal (LLNs) for the FEV1/FVC ratio was provided by the Third National Health and Nutrition Examination Survey (NHANES III), in which a ratio less that the fifth percentile of the distribution of Z-scores correctly identified persons with an increased risk of death and prevalence of respiratory symptoms [7].

Data from Wave 2 of the UK Household Longitudinal Survey and the Health Survey for England 2010 were used to determine the prevalence of chronic airflow obstruction in 7879 participants aged 4095 years without diagnosed asthma and good-quality spirometry data [8]. Three definitions were applied: self-reported physician-diagnosed COPD; an FEV1/FVC cut-point of 0.7; and an age-, sex-, height- and ethnicity-specific LLN of the FEV1/FVC ratio. Of those studied, 22.2\% had chronic airflow obstruction according to the fixed ratio cut-point, compared with $13.1 \%$ when using LLN criteria and, remarkably, only $2.8 \%$ reported physician-diagnosed COPD.

In this issue of the European Respiratory Journal, AANERUd et al. [9] present data from the general-population-based European Community Respiratory Health Survey (ECRHS) to address the impact of smoking on the development of chronic airflow obstruction defined by FEV1/FVC $<0.7$ in the presence of known pre-existing airway disease. Data from over 15000 persons aged 20-56 years were

Received: Dec 172014 | Accepted: Dec 182014

Conflict of interest: None declared.

Copyright OERS 2015 
examined, including almost 9000 who underwent spirometry in both the initial phase of ECRHS and in a follow-up assessment 9 years later. A significant limitation of the study is that only pre-bronchodilator data were available. Nonetheless, there are several pertinent findings adding to the current knowledge of the effects of smoking on asthma and chronic airflow obstruction.

Firstly, and not surprisingly, smoking is associated with the development of chronic airflow obstruction. Among subjects without asthma, current smokers had a 4.5 times higher risk of airflow obstruction compared with never-smokers. The actual prevalence rates of airflow obstruction among those with no asthma were low in this relatively young age group: $0.3 \%$ in nonsmokers and $1.5 \%$ in smokers. In a somewhat older population (NHANES III), VOLLMER [10] reported a prevalence of airflow obstruction, age adjusted to all study participants, of $25.8 \%$ in current smokers versus $8.1 \%$ in never-smokers, again using the fixed FEV1/FVC ratio of 0.7 as the definition. In 40-95-year-olds in the UK, the prevalence of airflow obstruction among smokers was $37.0 \%$ and among nonsmokers was $14.7 \%$ using FEV1/FVC $<0.7$, but substantially lower (29.8\% versus $6.8 \%)$ using LLN criteria [8].

Secondly, early-onset asthma is associated with the development of chronic airflow obstruction independent of smoking. Those with asthma with an onset before age 10 years had a 20 -fold increase in the prevalence of adult airflow obstruction, independent of smoking status (never smokers: OR 21.0, 95\% CI 12.7-35; current smokers: OR 23.7, 95\% CI 13.9-40.6). These results are consistent with other data showing that asthma per se can lead to chronic airflow obstruction mimicking COPD. Study members followed in a longitudinal birth cohort in Dunedin, New Zealand, had lung function measurements available from the age of 9 years into adult life [11]. Those with persistent asthma at the age of 26 years showed significant airflow obstruction, with an FEV1/FVC ratio $9-10 \%$ lower than their healthy peers in the cohort. Tracking their lung function backwards in time revealed that this degree of airflow limitation had been consistently present from the age of 9 years and the slope of the decline suggested it began much earlier in childhood. At the age of 38 years, almost $9 \%$ of Caucasians in this population-based, non-high risk cohort had a degree of fixed airflow obstruction, with a post-bronchodilator $\mathrm{FEV}_{1 / \mathrm{FVC}}$ ratio more than two standard deviations below the mean ratio for nonasthmatic, nonsmoking study members [12].

Several longitudinal studies confirm that the initial severity of asthma is a major factor predicting outcomes of persistence and impaired lung function in adult life [13]. Many children with moderate-to-severe asthma are now adults with persistent airflow obstruction and a degree of irreversibility. In population studies, these individuals are sometimes classified as having COPD based solely on their $\mathrm{FEV}$ 1/FVC ratio [14], which is inappropriate when there is a history of asthma [15].

Thirdly, smoking interacts with asthma, increasing the risk of chronic airflow obstruction. Among those with onset of asthma after the age of 10 years, the association with adult airflow obstruction was stronger among current smokers (a 25-fold increase) than never-smokers (an 11-fold increase). Among nonatopic asthmatics, the risk almost tripled from $5.6 \%$ to $14.9 \%$, whereas in atopic asthmatics, the risk increased from $7.1 \%$ to $11.8 \%$, which although not statistically significant, is still a $60 \%$ increase in risk. Does atopy partially protect against the impact of smoking, or is the airway of the atopic asthmatic already chronically inflamed such that the damaging effects of cigarette smoke are less evident [16]? As noted by the authors, atopy itself has been associated with accelerated decline in lung function in subjects with and without asthma [17].

Finally, AANERUD et al. [9] suggest that the adverse effects of smoking by an asthmatic may be synergistic and not just additive. Individuals with early-onset asthma had a 20 -fold increased risk of chronic airflow obstruction, but no detectable relative excess risk due to an interaction of current smoking and early-onset asthma. However, in those with later-onset asthma, there was a 12-fold increased relative excess risk of airway obstruction due to an interaction between current smoking and late-onset asthma.

It is clear that persons with asthma may develop chronic airflow obstruction and have similar lung function to those with classical COPD. SEXTON et al. [18] undertook a cross-sectional case-comparison study of New Zealand smokers and nonsmokers aged over 45 years with "COPD" based on spirometric criteria of FEV1/ FVC $<0.7$. Smokers had more severe airflow obstruction but the prevalence of respiratory symptoms was similar between the groups. Asthma was nearly universal among the nonsmokers and reported to be "the commonest identifiable cause of COPD" in that group. Nonsmokers had greater rates of atopy, and elevated exhaled nitric oxide and eosinophil counts, all consistent with asthma. Similarly, AL-KASsimi et al. [19] studied 80 patients in Saudi Arabia aged 40-70 years with $>10$ pack-years smoking history who fulfilled the spirometric criteria for COPD, with post-bronchodilator FEV1/FVC $<0.7$ and FEV1 $<80 \%$ predicted. The initial diagnosis was COPD in 46 (57.5\%) patients and asthma in 34 (42.5\%). After detailed evaluation, the final classification was steroid-responsive asthma in $48(60 \%)$ patients, irreversible asthma in eight (10\%), COPD in only 16 (20\%) and unclassifiable airflow limitation in eight (10\%). 
The term asthma-COPD overlap syndrome (ACOS) is increasingly being applied to patients with airflow obstruction related to smoking who have features of asthma (reversibility, eosinophilia and atopy) or who have a known past history of asthma. In the COPDGene study, subjects who had had asthma before the age of 40 years and also had at least 10 pack-years of smoking exposure had more exacerbations, less emphysema and more airway disease for any degree of lung function impairment compared with those with COPD alone, suggesting asthma drives both symptoms and pathology in these "ACOS" subjects [20].

The interplay between asthma and smoking, and impact on chronic airflow obstruction have been examined in the Tasmanian Longitudinal Health Study cohort [21]. Follow-up in adulthood (age 4144 years) found, after reweighting to the population, $6.0 \%$ had chronic airflow obstruction defined as a post-bronchodilator FEV1/FVC ratio below the LLN. The association with early-onset (reported at the ages of 7 or 14 years), current (symptoms in last 12 months) clinical asthma was equivalent to 33 pack-years of smoking, compared with 24 pack-years for later-onset (reported after the age of 20 years), current clinical asthma.

Placing the study of AANERUd et al. [9] in the context of existing literature, what can we conclude? Two key messages are that persistent asthma can be associated with reduced lung function, and that chronic airflow obstruction in adulthood even in smokers should not be considered COPD without giving consideration to earlier asthma. However, smoking does adversely impact lung function in asthmatics, perhaps by adding another airway disease, or by worsening existing asthma.

\section{References}

1 Vignoud L, Pin I, Boudier A, et al. Smoking and asthma: disentangling their mutual influences using a longitudinal approach. Respir Med 2011; 105: 1805-1814.

2 Halbert RJ, Natoli JL, Gano A, et al. Global burden of COPD: systematic review and meta-analysis. Eur Respir J 2006; 28: 523-532.

3 Johannessen A, Omenaas ER, Bakke PS, et al. Implications of reversibility testing on prevalence and risk factors for chronic obstructive pulmonary disease: a community study. Thorax 2005; 60: 842-847.

4 Quanjer PH, Enright PL, Miller MR, et al. The need to change the method for defining mild airway obstruction. Eur Respir J 2011; 37: 720-722.

5 Swanney MP, Ruppel G, Enright PL, et al. Using the lower limit of normal for the FEV1/FVC ratio reduces the misclassification of airway obstruction. Thorax 2008; 63: 1046-1051.

6 Cerveri I, Corsico AG, Accordini S, et al. Underestimation of airflow obstruction among young adults using FEV1/ FVC $<70 \%$ as a fixed cut-off: a longitudinal evaluation of clinical and functional outcomes. Thorax 2008; 63: $1040-1045$

7 Vaz Fragoso CA, Concato J, McAvay G, et al. The ratio of FEV1 to FVC as a basis for establishing chronic obstructive pulmonary disease. Am J Respir Crit Care Med 2010; 181: 446-451.

8 Scholes S, Moody A, Mindell JS. Estimating population prevalence of potential airflow obstruction using different spirometric criteria: a pooled cross-sectional analysis of persons aged 40-95 years in England and Wales. BMJ Open 2014; 4: e005685.

9 Aanerud M, Carsin A-E, Sunyer J, et al. Interaction between asthma and smoking increases risk of adult airway obstruction. Eur Respir J 2015; 45: 635-643.

10 Vollmer WM. Epidemiology of COPD: overview and the US perspective. Eur Respir J 2003; 22: Suppl. 43, 1s-3s.

11 Sears MR, Greene JM, Willan AR, et al. A longitudinal, population-based, cohort study of childhood asthma followed to adulthood. $N$ Engl J Med 2003; 349: 1414-1422.

12 Belsky DW, Sears MR, Hancox RJ, et al. Polygenic risk and the development and course of asthma: an analysis of data from a four-decade longitudinal study. Lancet Respir Med 2013; 1: 453-461.

13 Tai A, Tran H, Roberts M, et al. Outcomes of childhood asthma to the age of 50 years. J Allergy Clin Immunol 2014; 133: 1572-1578.

14 Shirtcliffe $\mathrm{P}$, Weatherall $\mathrm{M}$, Marsh $\mathrm{S}$, et al. COPD prevalence in a random population survey: a matter of definition. Eur Respir J 2007; 30: 232-239.

15 Sears MR. Population prevalence of COPD. Eur Respir J 2008; 31: 218.

16 Polosa R, Thomson NC. Smoking and asthma: dangerous liaisons. Eur Respir J 2013; 41: 716-726.

17 Weiss ST. Atopy as a risk factor for chronic obstructive pulmonary disease: epidemiological evidence. Am J Respir Crit Care Med 2000; 162: S134-S136.

18 Sexton P, Black P, Wu L, et al. Chronic obstructive pulmonary disease in non-smokers: a case-comparison study. COPD 2014; 11: 2-9.

19 Al-Kassimi FA, Abba AA, Al-Hajjaj MS, et al. Asthma masquerading as chronic obstructive pulmonary disease: a study of smokers fulfilling the GOLD definition of chronic obstructive pulmonary disease. Respiration 2011; 82: $19-27$.

20 Hardin M, Cho M, McDonald M-L, et al. The clinical and genetic features of COPD-asthma overlap syndrome. Eur Respir J 2014; 44: 341-350.

21 Perret JL, Dharmage SC, Matheson MC, et al. The interplay between the effects of lifetime asthma, smoking, and atopy on fixed airflow obstruction in middle age. Am J Respir Crit Care Med 2013; 187: 42-48. 